\title{
On an approximate construction for a regular polygon
}

\author{
By S. A. Scotт.
}

My attention was drawn by an Art teacher to the following approximate construction ${ }^{1}$ for inscribing a regular polygon of $n$ sides in a given circle, having diameter $A B$, centre $O$. Find $C$ in $A B$ so that $A C: A B=2: n$, and construct the equilateral triangle $A B D$. If $D C$ produced meets the circle in $E$, then $A E$ is approximately a side of the required polygon.

I have found ${ }^{2}$ that the value of $\tan A O E$, as given by this construction, is $\left(\sqrt{3 n^{2}+48 n-96}-\sqrt{3 n^{2}}\right) /(2 n-8)$, from which the values tab ulated below were calculated. For large $n$, the expansion of this expression begins with the terms $\sqrt{3}\left(4 n^{-1}-8 n^{-2}+\ldots\right)$ as compared with $\tan 2 \pi / n=2 \pi n^{-1}+\frac{8}{3} \pi^{3} n^{-3}-\ldots$ Evidently the construction becomes increasingly erroneous as $n$ increases: the limiting percentage error is about $10 \cdot 3 \%$. Yet when $n$ is not large it is surprisingly accurate, and it happens to be precise for $n=2,3,4$ or 6 .

\begin{tabular}{rccccccccc}
$n:$ & 3 & 4 & 5 & 6 & 7 & 8 & 10 & 20 & 60 \\
$360^{\circ} / n:$ & $120^{\circ}$ & $90^{\circ}$ & $72^{\circ}$ & $60^{\circ}$ & $51^{\circ} 26^{\prime}$ & $45^{\circ}$ & $36^{\circ}$ & $18^{\circ}$ & $6^{\circ}$ \\
$A O E:$ & $120^{\circ}$ & $90^{\circ}$ & $71^{\circ} 57^{\prime}$ & $60^{\circ}$ & $51^{\circ} 31^{\prime}$ & $45^{\circ} 11^{\prime}$ & $36^{\circ} 21^{\prime}$ & $18^{\circ} 38^{\prime}$ & $6^{\circ} 26^{\prime}$ \\
$\%$-error: & 0 & 0 & -0.07 & 0 & $0 \cdot 17$ & 0.41 & $0 \cdot 97$ & $3 \cdot 5$ & $7 \cdot 2$. \\
\hline
\end{tabular}

1 I. H. Morris, Geometrical Drawing for Art Students, p. 40; Longmans Green \& Co.

2 The author has supplied two proofs, using coordinate and trigonometrical methods. Readers, or their pupils, may find this an interesting exercise.-Editor.

49 Sicily Park, Finaghy, Belfast. 